\title{
Improving the accuracy of a time lens
}

\author{
Vladimir B. Yurchenko \\ Department of Electrical and Electronics Engineering, Bilkent University, Bilkent, Ankara TR-06533, Turkey, \\ and Institute of Radiophysics and Electronics, National Academy of Sciences of Ukraine, \\ 12 Proskura Street, Kharkov 310085, Ukraine
}

Received February 11, 1997; revised manuscript received July 22, 1997

\begin{abstract}
A method for improving the accuracy of temporal imaging with an imperfect time lens is proposed. Signal distortion arising from complicated dispersion of the delay lines can be reduced considerably by appropriate choice of the phase-modulation function including the second harmonic of the basic modulation frequency and a specific phase shift of the modulation with respect to the main signal. The method is of particular interest for picosecond and femtosecond optical pulse generation. (C) 1997 Optical Society of America [S0740-3224(97)05211-9]
\end{abstract}

\section{INTRODUCTION}

Recently there has been considerable interest in temporal imaging with a time lens. ${ }^{1-3}$ A time lens can stretch and compress signals in time by varying phase relations among various components of their spectra. ${ }^{1}$ The idea of transforming signal spectra to resolve and compress signals was introduced in radiophysics ${ }^{4}$ and later developed in optics with the purpose of generating ultrashort laser pulses. $^{5-8}$

The action of the time lens has been thoroughly treated by Kolner and Nazarathy ${ }^{1}$ and by Kolner. ${ }^{2,3}$ It is based on the analogy between the propagation of a wave packet in a dispersive medium and the diffraction of a beam in free space. Spatial imaging with a glass lens involves diffraction from object to lens, a spatially dependent phase shift provided by the lens, and diffraction from the lens to an image. Similarly, temporal imaging with a time lens requires dispersion from an object pulse to the lens, a time varying phase shift that is due to the lens, and dispersion from the lens to an image pulse. Hence the entire time lens system consists of two dispersive elements (delay lines) and a phase modulator between them (the time lens itself). In the research reported in Refs. 7 and 8, dispersive elements were realized by grating pairs, and the optical phase modulator was composed of a $\mathrm{LiNbO}_{3}$ crystal placed in a waveguide with the phase velocity of the driving microwave mode matched to that of the optical wave in the crystal. Similarly to its spatial counterpart, the time lens can rescale temporal signals, preserving their shape, when some special tuning conditions are satisfied (see below).

The time lens is perfect when the phase shift that arises in the dispersive lines is quadratic in frequency and the phase modulation is quadratic in time. In this case, the initial signal is scaled in time, with no distortion if the parameters of the functions $\beta(\omega)$ and $\varphi(t)$, where $\beta$ is the propagation constant, $\omega$ is the frequency, $\varphi$ is the phase modulation, and $t$ is the time, are matched according to the equation of a lens. ${ }^{1,2}$ This is the case that was carefully studied in previous papers. ${ }^{1-3}$

In practice, the time lens is not perfect because the phase modulation is normally sinusoidal ${ }^{7,8}$ and the dispersion law is not exactly quadratic. Therefore some signal distortion is inevitable and is often more significant than the regular nonparaxial effects in the spatial lens. In the time lens, however, there is a possibility of controlling not only the phase modulation $\varphi(t)$ but both functions $\varphi(t)$ and $\beta(\omega)$ simultaneously. In this way one can try to adjust the functions properly to decrease signal distortion to a minimum even when both of them are not quadratic.

We propose a method for improving the accuracy of temporal imaging with an imperfect time lens by optimum choice of phase modulation adjusted to the given nonquadratic dispersion of the delay lines used in the system.

\section{FORMULATION}

In the dispersive medium the wave equation for the electric field $E(t, z)$ is of the form

$$
\frac{\partial^{2} E(t, z)}{\partial z^{2}}-\frac{1}{c^{2}} \frac{\partial^{2}}{\partial t^{2}} \int_{0}^{\infty} \varepsilon_{r}(\tau) E(t-\tau, z) \mathrm{d} \tau=0,
$$

where $\varepsilon_{r}(\tau)$ is the relative dielectric function of the medium and $c$ is the speed of light in free space. One can find an analytical solution to Eq. (1) by using the Laplace transform, which is the most suitable method for the given problem. ${ }^{9}$ In terms of the envelope of the wave packet $E(t, z)=U(t, z) \exp \left\{i\left[\omega_{0} t-\beta\left(\omega_{0}\right) z\right]\right\}$ at the carrier frequency $\omega_{0}$, the solution is

$$
U_{1}(\theta, z)=\int_{-\infty}^{\infty} U_{0}(t) G(\theta-t, z) \mathrm{d} t,
$$

where $U_{0}(t)=U(t, 0)$ is the input signal, $U_{1}(\theta, z)$ is the signal at a distance $z, \theta=t_{1}-z / v_{g}, v_{g}$ is the group velocity at frequency $\omega_{0}, G(\theta-t, z)$ is the Green's function given by the integral

$$
G=\frac{1}{2 \pi} \int_{-\infty}^{\infty} \exp \left\{i\left[(\theta-t)\left(\omega-\omega_{0}\right)-\Gamma(\omega) z\right]\right\} \mathrm{d} \omega,
$$


$\Gamma(\omega)=\beta(\omega)-\beta_{0}-\beta_{1}\left(\omega-\omega_{0}\right), \quad \beta^{2}(\omega)=\varepsilon_{r}(\omega) \omega^{2} / c^{2}$, $\varepsilon_{r}(\omega)$ is the Fourier transform of the dielectric function $\varepsilon_{r}(\tau), \beta_{0}=\beta\left(\omega_{0}\right)$, and $\beta_{1}=1 / v_{g}=\partial \beta(\omega) /\left.\partial \omega\right|_{\omega=\omega_{0}}$.

In the third-order approximation, when

$$
\begin{aligned}
\beta(\omega)= & \beta_{0}+\beta_{1}\left(\omega-\omega_{0}\right)+1 / 2 \beta_{2}\left(\omega-\omega_{0}\right)^{2} \\
& +1 / 6 \beta_{3}\left(\omega-\omega_{0}\right)^{3},
\end{aligned}
$$

one has

$$
\begin{aligned}
G= & G_{3}(\theta-t, z)=\frac{1}{2 \pi} \int_{-\infty}^{\infty} \exp \left\{i \left[(\theta-t) \omega-1 / 2 \beta_{2} z \omega^{2}\right.\right. \\
& \left.\left.-1 / 6 \beta_{3} z \omega^{3}\right]\right\} \mathrm{d} \omega .
\end{aligned}
$$

In the second order, when $\beta_{3}=0, G(\theta-t, z)$ is simplified to

$$
G=G_{2}(\theta-t, z)=\left(2 \pi i \beta_{2} z\right)^{-1 / 2} \exp \left[i(\theta-t)^{2} / 2 \beta_{2} z\right],
$$

providing an exactly quadratic phase shift in the frequency domain.

Consider now the action of the time lens. Suppose that the dispersive lines are of lengths $L_{1}$ and $L_{2}$, and that the phase modulator transforms the signal $U_{1}\left(\theta, L_{1}\right)$ as

$$
U_{2}\left(\theta, L_{1}\right)=U_{1}\left(\theta, L_{1}\right) \exp [i \varphi(\theta)],
$$

where $\varphi(\theta)=\varphi_{0}+\varphi_{1} \theta+\varphi_{2} \theta^{2}+\varphi_{3} \theta^{3}$ in the thirdorder approximation. Then the output signal $U_{3}(\tau)$ at the end of the system is given by the relation

$$
\begin{aligned}
U_{3}(\tau)= & \int_{-\infty}^{\infty} \mathrm{d} t U_{0}(t) \int_{-\infty}^{\infty} \mathrm{d} \theta G\left(\tau-\theta, L_{2}\right) \\
& \times \exp [i \varphi(\theta)] G\left(\theta-t, L_{1}\right),
\end{aligned}
$$

where $\tau=\theta-\left(z-L_{1}\right) / v_{g}$.

When $G=G_{2}$ and the phase modulation is quadratic, $\varphi(\theta)=\varphi_{2} \theta^{2}$, one obtains

$$
\begin{aligned}
U_{3}(\tau)= & \frac{-i}{\beta_{2} \sqrt{L_{1} L_{2}}} \exp \left(\frac{i \tau^{2}}{2 \beta_{2} L_{2}}\right) \int_{-\infty}^{\infty} \mathrm{d} t U_{0}(t) I(t, \tau) \\
& \times \exp \left(\frac{i t^{2}}{2 \beta_{2} L_{1}}\right)
\end{aligned}
$$

where

$$
\begin{aligned}
I(t, \tau)= & \frac{1}{2 \pi} \int_{-\infty}^{\infty} \mathrm{d} \theta \exp \left[i \theta^{2}\left(\frac{1}{2 \beta_{2} L_{2}}+\varphi_{2}+\frac{1}{2 \beta_{2} L_{1}}\right)\right] \\
& \times \exp \left[-i \frac{\theta}{\beta_{2}}\left(\frac{\tau}{L_{2}}+\frac{t}{L_{1}}\right)\right] .
\end{aligned}
$$

In this case, under the condition that

$$
1 /\left(2 \beta_{2} L_{2}\right)+1 /\left(2 \beta_{2} L_{1}\right)+\varphi_{2}=0,
$$

which is the regular equation of a lens, ${ }^{1}$ one has $I(t, \tau)$ $=\beta_{2} L_{1} \delta\left(t+\tau L_{1} / L_{2}\right)$ and obtains the well-known result ${ }^{10}$ that the output signal $U_{3}(\tau)$ is of the same form as the input signal but of a different time scale, i.e.,

$$
U_{3}(\tau)=-i\left(\frac{L_{1}}{L_{2}}\right)^{1 / 2} \exp \left(i \tau^{2} \frac{L_{1}+L_{2}}{2 \beta_{2} L_{2}^{2}}\right) U_{0}\left(-\frac{L_{1}}{L_{2}} \tau\right),
$$

where the scaling factor is $K^{(0)}=-L_{1} / L_{2}$ (the phase factor is not essential).

When the dispersion law $\beta(\omega)$ and the phase modulation $\varphi(\theta)$ are not quadratic, the inner integral in the general formula [Eq. (8)] is not simplified to the $\delta$ function. Therefore, in this case, the output signal $U_{3}(\tau)$ is no longer similar to $U_{0}(t)$. The exact analysis of the transform is rather complicated, even in the third-order approximation, when $G=G_{3}$ according to Eq. (5). However, if one takes $G_{3}$ as

$$
G_{3}=G_{3}(\theta-t, z)=A(\theta-t, z) \exp [i F(\theta-t, z)],
$$

and makes a notion that, in the given phenomenon, variation of the phase factor $F(\theta-t, z)$ compared with $A(\theta$ $-t, z)$ is more essential, an approximate analysis is possible.

Equation (13) arises naturally if the stationary-phase asymptotic evaluation of integral (5) is performed. If a few points of the stationary phase exist (e.g., two, as for the function $G_{3}$ ), one can for simplicity take into account only one of them, which relates to the minimum oscillations in function (13) to yield the main contribution to integral (8).

Let us expand phase function $F_{i}$ ( $i=1,2$ refers to the first or the second dispersion line, respectively) in the power series in $\theta-t$ (or $\tau-\theta$ ) with descending coefficients $f_{i k}$. When $i=1$, one has, for example, $F_{1}(\theta$ $\left.-t, L_{1}\right)=f_{10}+f_{11}(\theta-t)+f_{12}(\theta-t)^{2}+f_{13}(\theta-t)^{3}$, where the third-order approximation is used. Then Eq (8) takes the form

$$
U_{3}(\tau)=\int_{-\infty}^{\infty} \mathrm{d} t U_{0}(t) \int_{-\infty}^{\infty} \mathrm{d} \theta C(\theta, t, \tau) \exp [i S(\theta, t, \tau)],
$$

where

$$
\begin{aligned}
S(\theta, t, \tau)=\theta[ & \left(f_{11}-f_{21}+\varphi_{1}\right)-2\left(f_{12} t+f_{22} \tau\right) \\
& \left.+3\left(f_{13} t^{2}-f_{23} \tau^{2}\right)\right]+\theta^{2}\left[\left(f_{12}+f_{22}+\varphi_{2}\right)\right. \\
& \left.-3\left(f_{13} t-f_{23} \tau\right)\right]+\theta^{3}\left[f_{13}-f_{23}+\varphi_{3}\right] .
\end{aligned}
$$

If the stationary-phase method that accounts for one stationary point is applied to Eq. (5), the coefficients $f_{i k}$ are found approximately as $f_{i 1}=0, f_{i 2}=1 /\left(2 \beta_{2} L_{i}\right)$, and $f_{i 3}=\beta_{3} /\left(6 \beta_{2}{ }^{2} L_{i}{ }^{2}\right)$. Analysis shows, however, that for accurate signal scaling more careful evaluation is required, and the phase $F(\theta-t, z)$ in Eq. (13) should be taken as a mean-value function that smooths the actual oscillating phase of the original Green's function $G_{3}(\theta$ $-t, z)$ given by Eq. (5); see the numerical example and Figs. 1 and 2 below.

Now, by applying the stationary-phase method to the inner integral in Eq. (14), one obtains the output signal $U_{3}(\tau)$ as

$$
\begin{aligned}
U_{3}(\tau)= & \int_{-\infty}^{\infty} \mathrm{d} t U_{0}(t)\left\{C_{1}\left(\theta_{1}\right) \exp \left[i S\left(\theta_{1}\right)\right]\right. \\
& \left.+C_{2}\left(\theta_{2}\right) \exp \left[i S\left(\theta_{2}\right)\right]\right\}
\end{aligned}
$$


where $\theta_{1}=\theta_{1}(t, \tau)$ and $\theta_{2}=\theta_{2}(t, \tau)$ are two different points of the stationary phase given by the equation

$$
\partial S(\theta, t, \tau) / \partial \theta=0
$$

As one can see, the output signal $U_{3}(\tau)$ is not similar to the input signal, which means a certain distortion of the signal by the time lens [when $f_{13}=f_{23}=\varphi_{3}=0$, the inner integral in Eq. (14) is a $\delta$ function, which reduces Eq. (14) to Eq. (12)].

However, noting the small values of the coefficients $f_{i 3}$ and taking them into account in the first power only, one can considerably simplify Eqs. (16) and (17). In this case, when the following three conditions are satisfied:

$$
\begin{aligned}
& f_{11}-f_{21}+\varphi_{1}=0 \\
& f_{12}+f_{22}+\varphi_{2}=0 \\
& f_{13}-f_{23}+\varphi_{3}=0,
\end{aligned}
$$

the phase function $S(\theta, t, \tau)$ has only one stationary point, integral equation (16) can be further analyzed in a similar way, and finally, when some small additional amplitude variations are neglected, the output signal can be written, approximately, in the form

$$
U_{3}(\tau)=U_{0}\left[t_{0}(\tau)\right] \exp \left\{i T\left[t_{0}(\tau)\right]\right\} .
$$

A remarkable thing about Eq. (21) is that the function $t_{0}(\tau)$ is linear:

$$
t_{0}(\tau)=-\left(f_{22} / f_{12}\right) \tau,
$$

and is exactly of the same form that provides zero argument to the $\delta$ function that reduces Eq. (9) to Eq. (12). As a result, the output signal $U_{3}(\tau)$ is again similar to the input signal, $U_{0}(t)$.

\section{DISCUSSION}

Equations (18), (19), and (20) can be interpreted as the first-, the second-, and the third-order equations of a lens, respectively, with the coefficient $K=-f_{22} / f_{12}$ being the scaling factor of temporal imaging (the signal is compressed when $|K|>1$ ). Although the requirements of Eqs. (18)-(20) are approximate (as is the signal scaling that is performed), the equations work fairly well for phase-modulation tuning, as confirmed by the computer simulations described below.

When either $f_{13}=f_{23} \neq 0$ (symmetrical imperfect lens) or $f_{13}=f_{23}=0$ (perfect lens), Eq. (20) yields $\varphi_{3}=0$; i.e., quadratic phase modulation is optimal. In general, however, the third-order term $\varphi_{3}=f_{23}-f_{13}$ is needed; i.e., one requires nonsymmetrical nonquadratic phase modulation to optimize signal imaging.

Equation (19) is equivalent to the regular lens law [Eq. (11)] that is used to adjust the focal length and to estimate the scaling factor $K$. In the second-order approximation one has $f_{12}=f_{12}{ }^{(0)}=1 /\left(2 \beta_{2} L_{1}\right), \quad f_{22}=f_{22}{ }^{(0)}$ $=1 /\left(2 \beta_{2} L_{2}\right)$, and $K=K^{(0)}=-L_{1} / L_{2}$. In general, however, $K \neq K^{(0)}$ because of the effect of the higherorder dispersion, which alters the values of $f_{12}$ and $f_{22}$ compared with those of $f_{12}{ }^{(0)}$ and $f_{22}{ }^{(0)}$ because the former values depend on the parameter $\beta_{3}$ in the dispersion law.

The more essential fact is that the frequency of the phase modulation $^{1,2}$ that is related to the coefficient $\varphi_{2}$ has to be adjusted according to the accurate value $\varphi_{2}$ $=-\left(f_{12}+f_{22}\right)$ given by the more general equation of a lens, Eq. (19), than by that of the regular law, Eq. (11). The latter is especially important for achieving the lowdistortion imaging consistent with Eqs. (18)-(20).

The analytical results obtained are confirmed by accurate numerical modeling. Figure 1 shows the results of the computer simulation that illustrates the effect. The curves in Fig. 1 were scaled when plotted to enable them to be compared easily. As one can see, the regular signal distortion is considerable, but accurate choice of the phase modulation helps to decrease the distortion greatly. The parameters $\varphi_{1}, \varphi_{2}$, and $\varphi_{3}$ of the cubic phase modu-

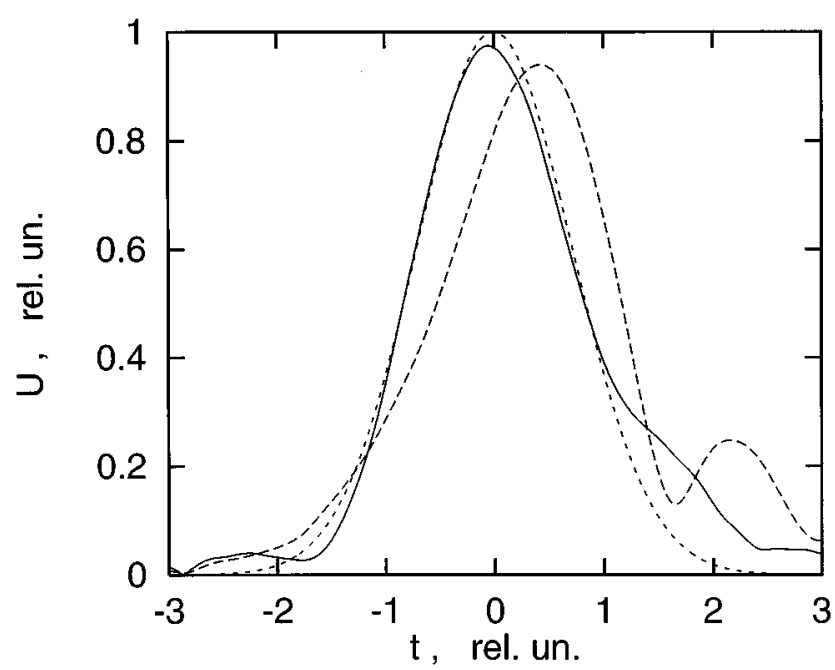

Fig. 1. Output signals $U_{3}$ computed with third-order phasematching (solid curve) and with typical quadratic phase modulation (longer-dashed curve), compared with the input signal $U_{0}(t)$ (shorter-dashed curve). The output signals scale back with the coefficients $K$ and $K^{(0)}$, respectively. The relevant parameters in relative units are $L_{1}=5, L_{2}=1, \beta_{0}=1, \beta_{1}=1, \beta_{2}=0.2$, $\beta_{3}=0.005$, which lead to $\varphi_{2}{ }^{(0)}=-3$ and $K^{(0)}=5$ for quadratic phase modulation, and $\varphi_{1}=0.03, \varphi_{2}=-3.08, \varphi_{3}=-0.12$, and $K=5.19$ for the third-order phase matching.

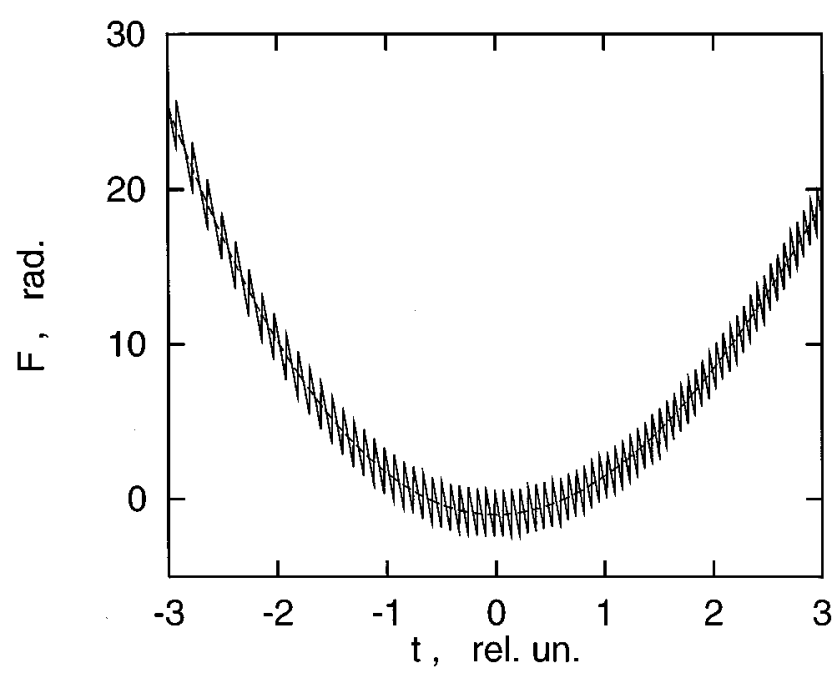

Fig. 2. Phase $F(t)$ of the Green's function $G\left(t, L_{2}\right)$ according to the stationary-phase method accounting for two stationary points (solid, jagged curve) and the third-order approximation of $F(t)$ (dashed, smooth curve) used to evaluate $\varphi(t)$. 
lation $\varphi(\theta)$ were determined by Eqs. (18)-(20) with the coefficients $f_{i k}$ obtained by approximating the phases of the Green's functions $G\left(\theta-t, L_{1}\right)$ and $G\left(\tau-\theta, L_{2}\right)$ computed accurately according to Eq. (5) (Fig. 2).

In practice, the accurate phase modulation required by Eqs. (18)-(20) can be introduced into the system when (a) the modulation running at some basic frequency $\omega_{m}$ is shifted in phase with respect to the main signal and (b) the second harmonic of the basic frequency $\omega_{m}$ is also used, so that the net modulation function is

$\varphi(\theta)=\left(2 \varphi_{2} / \omega_{m}{ }^{2}\right)\left[1-\delta \cos \left(\omega_{m} \theta+\alpha\right)-\gamma \sin \left(2 \omega_{m} \theta\right)\right]$,

where $\delta=\sqrt{1+\varepsilon}, \tan (\alpha)=\varepsilon, \varepsilon=\left(2 / 3 \omega_{m} \varphi_{1}+\varphi_{3} / \omega_{m}\right) /$ $\varphi_{2}$, and $\gamma=0.5\left(\varepsilon-0.5 \omega_{m} \varphi_{1} / \varphi_{2}\right)$. For the case shown in Fig. 1, this means that, at the maximum modulation frequency $\omega_{m}=0.1$ consistent with the signal duration, the relevant parameters are $\varepsilon=0.32, \delta=1.05$, and $\gamma$ $=0.16$, so the phase shift of the modulation with respect to the signal is $\sim \alpha=18^{\circ}$ and the relative amplitude of the second harmonic is nearly $16 \%$.

\section{CONCLUSION}

In conclusion, the results of this study have shown the possibility of increasing the accuracy of time imaging with an imperfect time lens by the proper choice of the phase-modulation function, depending on the dispersion law of the delay lines used in the lens.

\section{ACKNOWLEDGMENTS}

The author is grateful to K. A. Lukin for stimulating discussions. The research was supported by the State Committee on Science and Technology of Ukraine, project 2.3/ 827.

\section{REFERENCES}

1. B. H. Kolner and M. Nazarathy, "Temporal imaging with a time lens," Opt. Lett. 14, 630-632 (1989).

2. B. H. Kolner, "Generalization of the concepts of focal length and $f$-number to space and time," J. Opt. Soc. Am. A 11, 3229-3234 (1994).

3. B. H. Kolner, "Space-time duality and the theory of temporal imaging," IEEE J. Quantum Electron. 30, 1951-1953 (1994).

4. Ya. D. Shirman, Resolution and Compression of Signals (Soviet Radio, Moscow, 1974) (in Russian).

5. D. Grishkovskiy and A. C. Balant, "Optical pulse compression based on enhanced frequency chirping," Appl. Phys. Lett. 41, 1-3 (1982).

6. S. A. Akhmanov, V. A. Vysloukh, and A. S. Chirkin, "Selfaction of wave packets in a nonlinear medium and femptosecond laser pulse generation," Sov. Phys. Usp. 29, 642-677 (1987).

7. M. T. Kauffman, A. A. Godil, B. A. Auld, W. C. Banyai, and D. M. Bloom, "Applications of time lens optical systems," Electron. Lett. 29, 268-269 (1993).

8. A. A. Godil, B. A. Auld, and D. M. Bloom, "Time-lens producing 1.9 ps optical pulses," Appl. Phys. Lett. 62, 10471049 (1993).

9. S. J. Farlow, Partial Differential Equations for Scientists and Engineers (Wiley, New York, 1982).

10. J. W. Goodman, Introduction to Fourier Optics (McGrawHill, New York, 1968). 